\section{Problems in hominid taxonomy}

WE are pleased to have had our recent systematic assessment of hominid evolution recognised in Nature (News \& Views, 278, 400; 1979). Your Paleoanthropology Correspondent has, however, provided the readers of Nature with a misleading analysis of our published work as well as a misunderstanding of systematic procedures followed therein.

Your correspondent was not familiar with the authorship of the formal designation of the new taxon, Australopithecus afarensis, Johanson, White and Coppens, 1978 (not Johanson and White as reported). The correspondent also seems unaware of the difference between diagnosis and description in a systematic publication. Features of the dentition (large mandibular canines, threerooted upper premolars and molar size gradients) and postcranium (lack of third metacarpal styloid process, os coaxae anatomy) taken by your correspondent as diagnostic of $A$. afarensis are detailed in the description of the species and not the diagnosis. Of the suite of characters distinguishing $A$. afarensis, the correspondent mentions only a few dental features and completely neglects diagnostic cranial and mandibular anatomy

Unfortunately, treatment of our Science article $(203,321$; 1979) fares no better as several errors, in fact, mar the report. For instance, the correspondent reports that the Taung specimen does not have a $\mathrm{P}_{4}$ but that we measured it. In fact, the specimen has two $\mathrm{P}_{4} s$, but we did not measure them because they are unerupted. Similarly, our table of dental metrics (page 322) shows central incisor measurements for five individuals instead of the two reported by your correspondent. The correspondent also states that the $A$. afarensis $\mathrm{P}_{3}$ "has a large outer cusp and a very small inner cusp". The relevant citation from Science (page 322) is "A smaller lingual cusp is usually present, but some specimens (A.L. $288-1,128-23$ ) display only an inflated lingual ridge." The fact that this feature reminds the correspondent of Miocene hominoid $\mathrm{P}_{3} \mathrm{~s}$ emphasises our assertion that the $\mathrm{CP}_{3}$ complex of $A$. afarensis is an important character suite that allies the new taxon with extant and extinct apes and distingushes it from members of other hominid species.

Finally, the correspondent suggests that variation (not variability) in the Hadar sample is excessive and indicative of taxic diversity. We direct the reader to our Science paper where all of our metric and morphological comparisons of the Hadar and Laetolil samples with those of extant hominoids support the interpretation that the fossil hominid material is best considered a single, naturally variable, sexually dimorphic taxon.

Nature's policy during the controversy over the status of the Taung skull was "No notice is taken of anonymous communications". (Nature 116, 462; 1925) We favour that forgotten policy.

\section{DONALD JOHANSON}

Laboratory of Physical Anthropology

The Cleveland Museum of Natural

History, Cleveland, Ohio.

TIM D. WHITE

Department of Anthropology,

University of California,

Berkeley, California.

Our Correspondent RePlies: While I thank Johanson and White for their response to my recent News and Views comment I must point out that their reply does not effectively refute any of the major points of that comment. One of the major premises of that comment was that the diagnosis of the new species (Johanson, White \& Coppens Kirtlandia, 28, 1; 1978) does not adequately differentiate it from $A$. africanus, the closest comparative taxon. If that premise can be substantiated then the validity of $A$. afarensis, as a separate and distinct taxon, can be questioned. The necessity of a clearly stated differential diagnosis was noted by the authors when they quoted Mayr's imperative to "list the most important characters or character combinations that are peculiar to the giver taxon and by which it can be differentiated from other similar or closely related ones" (op. cit., page 8). Diagnoses in the general palaeontological literature show that most workers follow Mayr's dictum. Yet precise differential statements are missing from this diagnosis; those features which qualify $A$. afarensis as a separate species have not been made clear.

Moreover, characters which may have a high degree of taxonomic significance have been described differently in the original diagnosis and in a later publication. The size of the inner cusp of the $A$. afarensis $\mathrm{P}_{3}$ is a case in point. In the diagnosis of the new species it was stated that this feature "is often expressed only as (an) inflated lingual ridge ( $O P$. cit., page 6). In a later publication the "often expressed" ridge had become a "usually present" cusp (Johanson \& White Science 203, $322 ; 1979$ ). They cannot have it both ways and the fact that they have described the same important character state differently in two publications does not add strength to their taxonomic claims.

Further confusion in their work is apparent when, in their response, they claim that five individuals were used in obtaining measurements of the upper first incisor. Reference to my comment will show that part of that discussion centred on their claim that $A$. afarensis had broad upper first incisors. The breadth of this tooth is measured in the mesiodistal orientation and reference to their Table 1 (Science 203, 322; 1979) will show that four teeth were measured in this orientation, not five individuals (presumably yielding 10 teeth as they now inexplicably claim.

Moreover, Johanson and White suggest that 1 have confused, in my comment, the diagnosis of the new species with its description. Again, it is they who are confused. That stated that "certain traits . . (were) placed in the description due to the lack of comparative anatomical specimens from other species of Australopithecus". (Kirtlandia $288 ; 1979$ ). In my comment I pointed out that in characters of the hand, wrist and os coxae not only do comparative specimens exist for $A$. africanus but they are virtually identical in the two species.

The second major point of the News and Views comment did not concern the validity of the new taxon but centred instead on the number of taxa which may be contained in it. The authors acknowledge that the metric and morphological ranges may be broad in $A$. afarensis but they claim in the response that "all" of their comparisons between the ranges in the fossil sample and in living hominoids have supported attribution of the fossil material to a single taxon. They do indeed present (in footnotes) coefficients of variation for three features demonstrating that, in selected features, the range of variation in $A$. afarensis does not exceed that of living hominoids. Figure 6 which purportedly "clearly" (Science 203, 329, footnote 23) compares the range of variation for one $A$. afarensis feature with that of other hominoids is, in fact, an illustration of two distal femora.

In the end, however, the $A$. afarensis sample is an extremely important one and disputes over its taxonomic placement do not minimise that importance. Johanson and White are to be praised for their rapid publication of this material. phenomena, described by T. Chester (Caltech) have been observed from another dwarf nova $U$ Gem. He presented a technique for modelling these variations which preserves the phase of harmonics. Improved upper limits for higher harmonics of the pulses can be extracted.

The gas flow from a primary star to a compact body is complicated and poorly understood, making the interpretation of $\mathrm{X}$-ray spectra very difficuit. Spectral features from atomic transitions in iron and other elements have been observed in galactic X-ray sources. With the advent of more sensitive spectrometers, such as on the Einstein satellite, many more spectral features are being discovered. R. McCray (Colorado) showed how the analysis of spectral features will allow a determination of the physical conditions in, for example, the flow of accreting material near a compact object. The 'standard model' (appropriate to the solar corona) of an optically thin plasma, in collisional equilibrium, does not hold near to a compact body or in a supernova remnant. An alternative model, which is similar to that for a planetary nebula, is a central point X-ray source which photoionises its surroundings into highly excited states, even at low temperatures. Here, the Auger effect and fluorescence will be very important and radiative recombination lines may dominate the spectrum.

N. Shakura (Sternberg Astronomical Institute) has calculated the characteristic scale of turbulence in a disk which leads to an estimate of $\alpha$ (the ratio of the stress to the pressure in the outer part of a disk). His result, $\alpha \sim 10^{-3}$, differs significantly from previous estimates. Simulations using the precessing, twisted accretion disk model proposed to explain the 35 day cycle of $\mathrm{Her}$ X-1 which were presented by J. Petterson (University of Illinois) require $\alpha$ to be of order unity. This is not necessarily inconsistent with Shakura's work as $\alpha$ may vary throughout the disk. 